\title{
Facteurs Associés À L'usage Du Préservatif Dans Le Foyer Conjugal Chez Les Personnes Vivant Avec Le VIH Suivies Sur Un Site À Abidjan
}

\author{
Ayekoe Adou Ignace, MD \\ Gakoue Zadi Désiré, MD \\ Coulibaly Madikiny, MD \\ Djoman Christiane, MD
}

National Institute of Public Hygiene, Guinea

Ayekoe Davis Helmold Adou, Student

University Kofi Annan, Guinée Conakry, Guinea

Gourizro Kohou Sebastien, Social Worker

Yapo Chia Evelyne, Special Health Educator

Yao Kan Sylvère, Special Health Educator

N'guessan Kouadio Serge, Social Worker

National Institute of Public Hygiene, Guinea

Doi:10.19044/esj.2019.v15n36p230 URL:http://dx.doi.org/10.19044/esj.2019.v15n36p230

\section{Resume}

L'infection à VIH en Côte d'Ivoire constitue une épidémie généralisée. En 2017 la prévalence était estimée à $2.8 \%$ et les nouvelles infections atteignaient le nombre de 29800. La transmission hétérosexuelle est de loin la plus importante. Les facteurs associés à la pratique du sexe sans condom sont nombreux. A l'opposé, on ne connaît pas les facteurs qui favorisent l'usage régulier du préservatif dans le foyer conjugal. L'objectif de cette étude était de décrire les facteurs associés à l'usage régulier du préservatif dans la sexualité intraconjugale chez les patients infectés par le VIH et sous traitement ARV. Une enquête quantitative transversale descriptive a été menée sur 6 mois continus du $1^{\text {er }}$ décembre 2017 au 31 mai 2018 à l'INHP. Etaient enrôlées à tout venant, les personnes vivant avec le VIH suivies sur le site et ayant donné leur consentement écrit. Les variables étudiées ont été réparties en groupes de caractéristiques individuelles, de situation matrimoniale, de reproduction, de durée de suivi, de divulgation du statut, de comportement sexuel dans le foyer, d'acceptabilité du préservatif. L'analyse des données a été faite avec le logiciel EPI INFO Version 3.5.4/2012. Les fréquences et les proportions des variables ont été calculées et le degré de signification a été déterminé. 102 personnes de la file active sous traitement ARV ont été enrôlées. La moyenne d'âge était 40 
ans avec un âge médian de 39 ans. 55,9\% des patients étaient en union dont $63,6 \%$ vivaient toujours avec leur même partenaire depuis leur dépistage. $61,8 \%$ des patients ont partagé leur statut à leur partenaire sexuel. Les couples sérodifférents représentaient $39 \%$. Dans le foyer, les rapports sexuels continuent d'être pratiqués chez $72 \%$ des personnes. Le préservatif était utilisé régulièrement chez 53,5\% durant les 6 derniers mois. Le fait d'être célibataire ou d'être informé du statut partenaire ou d'avoir une différence de statut était plus associé à l'utilisation constante du préservatif ; cependant aucune variable n'est significativement liée à un usage régulier du préservatif. Les PVVIH qui sont éduquées au port de préservatif, l'introduisent dans leur relation sexuelle intraconjugale. L'usage du préservatif serait plutôt déterminé par les problèmes et les besoins des individus qui l'utilisent. La difficulté de son utilisation régulière résulterait de la complexité et de la variabilité de ces problèmes et besoins vécus ou ressentis par les usagers.

Mots clés : Usage Régulier Du Préservatif ; Sexualité Conjugale ; VIH/Sida

\section{Factors Associated with Condom Use in the Marital Home Among People Living with HIV Followed at a Site in Abidjan}

Ayekoe Adou Ignace, MD

Gakoue Zadi Désiré, MD

Coulibaly Madikiny, MD

Djoman Christiane, MD

National Institute of Public Hygiene, Guinea

Ayekoe Davis Helmold Adou, Student

University Kofi Annan, Guinée Conakry, Guinea

Gourizro Kohou Sebastien, Social Worker

Yapo Chia Evelyne, Special Health Educator

Yao Kan Sylvère, Special Health Educator

N'guessan Kouadio Serge, Social Worker

National Institute of Public Hygiene, Guinea

\section{Abstract}

HIV infection in Côte d'Ivoire is a generalized epidemic. In 2017 the prevalence was estimated at $2.8 \%$ and new infections were 29,800 . 
Heterosexual transmission is by far the most important. In contrast, the factors that promote regular condom use in the marital home are not known.The objective of this study was to describe the factors associated with consistent condom use in intramarital sexuality among PLWH infection and ARV treatment. A descriptive cross-sectional quantitative survey was conducted over 6 consecutive months from December 1st, 2017 to May 31st, 2018 at the INHP. All PLWH who had been followed up and gave their written consent were enrolled. The variables studied were divided into groups of individual characteristics, marital status, children's needs, duration of follow-up, disclosure of status, sexual behaviour in the home, condom acceptability.

The data analysis was done with the software Epi Info 3.5.4/2012. Frequencies and proportions of the variables were determined and the degree of significance have been determined. 102 PLWHIV treated with ARV were enrolled. The average age was 40 years with a median age of 39 years. $55.9 \%$ of patients were in a relationship, $63.6 \%$ of whom were still living with their same partner since their screening. $61.8 \%$ of people shared their status with their sexual partner. Serodiscordant couples accounted for $39 \%$. In the household, sexual intercourse continues to be practised in $72 \%$ of people. The condom was used regularly in $53.5 \%$ during the last 6 months. The condom was used regularly in $53.5 \%$. Being single or being informed of partner status or having a status difference was more associated with consistent condom use; however, no variable is significantly related to consistent condom use. People living with HIV who are educated to condom use, introduce it into their intramarital sex. But its regular use is peppered with many difficulties. . Condom use would rather be determined by the problems and needs of the individuals who use it. The difficulty of its regular use would result from the complexity and variability of these problems and needs experienced or felt by the users.

Keywords: Condom's Use, Household, Sexual Intercourse, HIV/AIDS

\section{Introduction}

L'épidémie de l'infection à VIH est de type généralisé en Côte d'Ivoire. En 2017, la prévalence nationale était à 2,9 \% et inégalement répartie ; 4,1\% chez les femmes contre 1,7\% chez les hommes (DIIS, 2018). Depuis 1985, l'année de la notification du premier cas de l'infection à VIH, les différents gouvernements qui sont succédés et avec le soutien des partenaires au développement ont développé plusieurs stratégies pour contrer l'évolution de l'épidémie afin d'éradiquer cette épidémie à l'horizon de 2030 comme le recommandait l'ONUSIDA (2017). Parmi ces stratégies, on peut citer la communication pour le changement de comportement, la prévention de la transmission du VIH de la mère à l'enfant, le Conseil et le dépistage, la 
lutte contre la discrimination et la stigmatisation, les Violences Basées sur le Genre (VBG), l'adoption de loi portant régime de prévention, de protection et de répression en matière de $\mathrm{VIH})$, le traitement antiretroviral à toute personne dépistée positive et la promotion du port des préservatifs.

Les patients suivis dans les centres de prise en charge sont conseillés sur le risque de transmission sexuelle du VIH et en reçoivent gratuitement une dotation de 12 préservatifs par mois. Cependant le nombre de nouvelles infections est encore élevé. En 2017, c'étaient 29.800 nouvelles infections notifiées dont la majorité par voie sexuelle (DIIS, 2018). Cette situation porte à croire que le sexe sans condom ne pourrait être abandonné. Le taux d'usage régulier du préservatif dans les foyers est méconnu. Les facteurs associés à la pratique du sexe sans condom sont nombreux. Les auteurs comme Kalichman et al., (2016) et Wagner et al., (2016) ont montré que les valeurs sociales comme la stigmatisation, la discrimination et la non divulgation du statut au partenaire sexuel ont un lien avec le sexe sans condom. En revanche, au sein du foyer conjugal, on ne connait pas véritablement les facteurs qui peuvent influencer l'usage régulier du préservatif.

L'objectif de cette étude était de décrire les facteurs associés à l'usage régulier du préservatif dans la sexualité intraconjugale chez les patients infectés par le VIH et suivis sur un site de prise en charge.

\section{Methode Et Materiel D'etude \\ ○ Type d'étude}

Une enquête transversale et descriptive pour une étude quantitative sur une période de 6 mois allant du $1^{\mathrm{er}}$ décembre 2017 au 31 mai 2018 a été conduite sur le site de prise en charge des personnes vivant avec le VIH de l'Institut National d'Hygiène Publique (INHP).

\section{○ Population d'étude}

- Critères d'inclusion

- Etaient inclus dans l'étude toute personne vivant avec le VIH enrôlée dans les soins VIH et qui accepte librement de répondre au questionnaire après lecture et signature de la fiche de consentement.

- Concernant la sélection des candidats, c'étaient les patients venus pour une visite médicale de suivi ou un renouvellement du traitement ARV, soit pour un bilan biologique de suivi ou pour d'autres soins de santé.

\section{- Critères d'exclusion}

$\checkmark$ Les patients n'ayant pas totalisé au moins 6 mois de suivi dans les soins VIH ;

$\checkmark$ Les patients dont leur état de santé ce jour ne leur permet pas d'être soumis au questionnaire (patients malades). 


\section{- Collecte des données}

- Mode de collecte

Les données ont été collectées à l'aide d'un questionnaire. Ce questionnaire a été administré aux patients lors des visites médicales de suivi par les assistants sociaux exerçant sur le site. Ces patients ont été interrogés une seule fois quel que soit le nombre de visites effectuées sur la période d'enquête.

Afin d'éviter les doublons d'enrôlement, le numéro du suivi du client faisait partie de l'identification du client sur le masque de saisi des données.

\section{- Variables étudiées}

Les variables étudiés ont été réparties en groupes de caractéristiques individuelles : âge, sexe ; situation matrimoniale : lien avec le partenaire; de reproduction : nombre d'enfants du patient, désir d'enfants, nombre d'enfants nés après le dépistage et insémination pour enfanter; de durée de suivi : nombre d'années de suivi dans les soins ; de divulgation du statut : partage du statut avec le partenaire sexuel, connaissance du statut sérologique du partenaire sexuel; de comportement sexuel dans le foyer : régularité du port du préservatif, continuité du rapport sexuel; d'acceptabilité du préservatif : perception du préservatif.

\section{- Analyse des données}

Les données ont été codifiées et saisies sur le logiciel Epi Info Version 3.5.4/2012. Les fréquences et les proportions des variables étudiées ont été déterminées par le même logiciel. Les proportions d'usage régulier et d'usage inconstant du préservatif ont été comparées l'aide d'un test du Chi-deux.

\section{○ Considérations éthiques}

L'enrôlement de chaque sujet a fait l'objet d'obtention de son consentement. Une fiche de consentement est lue et expliquée à chaque patient. Il approuveson adhésion à l'étude en signant le document.

L'anonymat de chaque participant était assuré par l'utilisation de code ne permettant pas leur identification lors de l'exploitation des données.

\section{○ Limites de l'étude}

- Méthode de sélection des participants.

Les sujets ont été enrôlés à tout venant mais pas en exhaustivité $54 \%$ de la file active (102 sur 189 patients actifs). Deux faits expliquaient cette situation :(i) Les rendez-de-vous de visite des patients sont le plus souvent long. Ils sont trimestriels pour certains et semestriels pour d'autres. (ii) Tous ceux qui viennent à leur visite ne pourraient être interrogés surtout pendant les jours de grandes affluences; uniquement ceux qui pouvaient accorder quelques minutes de patience, étaient interrogés par les 2 prestataires en charge de l'enquête. 
- Sincérité des déclarations

Les patients ont été interviewés sur le site par les assistants sociaux chez qui recevaient régulièrement des conseils de prévention pendant les séances de counseling. Ces résultats obtenus sur le port du préservatif pourraient être différents si ces patients étaient interrogés ailleurs par des personnes autres que leurs assistants sociaux.

\section{Resultats}

\section{Facteurs associés à l'usage du préservatif}

\subsection{Caractéristiques individuelles}

102 patients infectés par le VIH suivis sur le site ont participé à cette étude. L'âge des patients variait de 20 à 60 ans avec une moyenne de 40 ans. Les femmes représentaient $71,6 \%$ des patients enrôlés.

\subsection{Durée de suivi dans les soins}

La durée du suivi des patients dans les soins VIH s'étendait sur 13 années de 2005 à 2018 et $53 \%$ des patients sont dépistés il y avait moins de 4 ans.

\subsection{Situation matrimoniale et reproduction dans le foyer conjugal}

Les patients en union représentaient $55,9 \%$. Parmi eux $63,6 \%$ vivaient toujours avec leur même partenaire depuis leur dépistage. $64,4 \%$ des patients ont exprimé un désir d'enfants. Les patients qui n'ont pas eu d'enfants représentaient 23\%. Parmi ceux qui ont enfanté, 28,6\% ont eu leurs enfants après le dépistage VIH. Et parmi ceux qui ont donné naissance à des enfants après le dépistage, aucun n'a eu recours à une insémination artificielle.

\subsection{Partage du statut sérologique dans le foyer conjugal}

$61,8 \%$ des patients ont partagé leur statut à leur actuel partenaire sexuel actuel et $64,7 \%$ des patients connaissaient le statut de leur partenaire sexuel. Les partenaires sexuels (lles) dont le statut est négatif (ves) atteignaient 39,2\% et positif $25,5 \%$.

\subsection{Comportement sexuel dans le foyer conjugal durant les 6 derniers mois}

1.5.1. Continuité des rapports sexuels dans le foyer conjugal durant les 6 derniers mois

Les rapports sexuels sont continuellement entretenus dans le foyer conjugal chez $72 \%$ des personnes interrogées durant les six derniers mois.

Les rapports sexuels sont arrêtés chez $28 \%$ des patients soit pour des raisons de veuvage, de maladie et désunion $(21 \%)$ ou soit pour des raisons personnelles $(7 \%)$. 
$21 \%$ des patients ont arrêté les rapports sexuels pour des raisons diverses (Veuvage, maladie) par contre $7 \%$ des patients.

\subsubsection{Usage du préservatif durant les 6 derniers mois dans le foyer conjugal}

Les patients ayant déclaré '"porter les préservatifs de façon régulière au cours des rapports sexuels durant les 6 derniers mois dans le foyer conjugal' ' représentaient $53,5 \%$.

\subsection{Acceptabilité du port du préservatif}

Ceux qui n'acceptaient pas ou ne toléraient pas l'usage du préservatif lors des rapports sexuels représentaient $21,2 \%$.

Les peines ressenties lors de l'usage du préservatif étaient principalement le manque de plaisir sexuel $(28,5 \%)$; la frustration $(33,3 \%)$ et l'anxiété, la colère, le découragement $(38,2 \%)$. Le tableau I présente les caractéristiques des variables étudiées.

Tableau I : Facteurs associés à l'usage du préservatif

\begin{tabular}{|c|c|c|c|c|}
\hline $\begin{array}{l}\text { Groupes de } \\
\text { facteurs }\end{array}$ & Variables & & Effectif (n) & Pourcentage \\
\hline \multirow{3}{*}{$\begin{array}{l}\text { Caractéristique } \\
\mathrm{s} \text { individuels }\end{array}$} & Sexe & Féminin & 73 & 71,6 \\
\hline & & Masculin & 29 & 28,4 \\
\hline & Age & $\begin{array}{l}\text { Moyenne d'âge } \\
\text { (année) }\end{array}$ & 40 & \\
\hline \multirow{6}{*}{$\begin{array}{l}\text { Situation } \\
\text { matrimoniale }\end{array}$} & & $\begin{array}{l}\text { Age médian } \\
\text { (année) }\end{array}$ & 39 & \\
\hline & & Mariage civil * & 16 & 15,7 \\
\hline & Vie maritale & Concubinage $* *$ & 41 & 40,2 \\
\hline & & Célibataire $* * *$ & 45 & 44,1 \\
\hline & Union avec le & Oui & 63 & 63,6 \\
\hline & $\begin{array}{l}\text { même } \\
\text { partenaire } \\
\text { depuis le } \\
\text { dépistage }\end{array}$ & Non & 36 & 36,4 \\
\hline \multirow{6}{*}{$\begin{array}{l}\text { Reproduction } \\
\text { des patients }\end{array}$} & $\begin{array}{l}\text { Reproduction } \\
\text { des patients }\end{array}$ & $\begin{array}{l}\text { Patients n'ayant } \\
\text { pas d'enfants }\end{array}$ & 23 & 23,0 \\
\hline & & $\begin{array}{l}\text { Patients ayant } \\
\text { un enfant }\end{array}$ & 26 & 26,0 \\
\hline & & $\begin{array}{l}\text { Patients ayant } \\
\text { plus d'un enfant }\end{array}$ & 51 & 51 \\
\hline & & $\begin{array}{l}\text { Patients ayant } \\
\text { d'enfants nés }\end{array}$ & & \\
\hline & & $\begin{array}{l}\text { après le } \\
\text { dépistage }\end{array}$ & 22 & 22,0 \\
\hline & $\begin{array}{l}\text { Insémination } \\
\text { artificielle }\end{array}$ & Oui & $\begin{array}{l}0 \\
101\end{array}$ & 100 \\
\hline
\end{tabular}


Non

\begin{tabular}{|c|c|c|c|c|}
\hline & Désir d'enfant & Oui & 62 & 64,6 \\
\hline & & Non & 34 & 35,4 \\
\hline Durée du suivi & Nombre & suivi il y a & 54 & 52,9 \\
\hline Dans les soins & d'années de & moins de 4 ans & & \\
\hline VIH & suivi dans les & & & \\
\hline & soins VIH & $\begin{array}{l}\text { Suivi il y a plus } \\
\text { de } 4 \text { ans }\end{array}$ & 48 & 47,1 \\
\hline & Statut & Positif & 26 & 25,5 \\
\hline & sérologique du & Négatif & 40 & 39,2 \\
\hline & partenaire & Nsp & 17 & 16,7 \\
\hline & sexuel & N/A (pas de & & \\
\hline $\begin{array}{l}\text { Partage du } \\
\text { statut }\end{array}$ & & $\begin{array}{l}\text { partenaire et pas } \\
\text { de rapport) }\end{array}$ & 19 & 18,6 \\
\hline & Partage du & Oui & 63 & 61,8 \\
\hline & statut avec le & Non & 20 & 19,6 \\
\hline & $\begin{array}{l}\text { partenaire } \\
\text { sexuel }\end{array}$ & $\begin{array}{l}\text { N/A (pas de } \\
\text { partenaire et pas } \\
\text { de rapport) }\end{array}$ & 19 & 18,6 \\
\hline & Usage du & Régulier & 54 & 53,5 \\
\hline & préservatif dans & Le plus souvent & 9 & 8,9 \\
\hline & les 6 derniers & Quelquefois & 15 & 14,9 \\
\hline & mois & Jamais & 7 & 6,9 \\
\hline $\begin{array}{l}\text { Comportement } \\
\text { sexuel }\end{array}$ & & $\begin{array}{l}\text { N/A (absence } \\
\text { ou arrêt de } \\
\text { rapport sexuel) }\end{array}$ & 16 & 15,8 \\
\hline & Continuité des & Non & 7 & 7,0 \\
\hline & rapports sexuels & $\begin{array}{l}\text { Non au début } \\
\text { mais ça va } \\
\text { maintenant }\end{array}$ & 7 & 7,0 \\
\hline & & $\begin{array}{l}\text { Oui mais pas } \\
\text { comme avant }\end{array}$ & 21 & 21,0 \\
\hline & & $\begin{array}{l}\text { Oui comme } \\
\text { d'habitude }\end{array}$ & 44 & 44,0 \\
\hline & & $\begin{array}{l}\text { N/A (Pas de } \\
\text { partenaire, arrêt } \\
\text { de rapport } \\
\text { sexuel) }\end{array}$ & 21 & 21,0 \\
\hline $\begin{array}{l}\text { Acceptabilité } \\
\text { du préservatif }\end{array}$ & $\begin{array}{l}\text { Acceptabilité/ } \\
\text { Tolérance du }\end{array}$ & Non & 21 & 21,2 \\
\hline & port de & Oui & 70 & 70,7 \\
\hline & préservatif & Sans avis & 8 & 8,1 \\
\hline
\end{tabular}




$\begin{array}{llll}\text { Perception du } & \text { Négative } & 23 & 22,8 \\ \text { port du } & \text { Positive } & 67 & 66,3 \\ \text { préservatif } & \text { Sans avis } & 11 & 10,9\end{array}$

* mariage civil : ce sont des partenaires sexuels qui sont dans un foyer dont le lien est reconnu par l'état civil.

* *concubinage : ce sont des partenaires sexuels qui sont en union dans un foyer dont le lien est autre que celui de l'état civil (dot, religion, etc.)

***célibataire regroupe ceux qui sont dans une relation libre (veuvage, désunion, sans partenaire fixe), mais reconnaissent un (une) partenaire sexuel avec qui, ils entretiennent des rapports sexuels.

\section{Lien des facteurs associés et l'usage régulier du préservatif dans les} rapports sexuels intraconjugaux

Les célibataires utilisaient régulièrement mieux le préservatif $(75,9 \%)$ que ceux qui sont en couple dans le foyer $(61,5 \%)$.

Les patients n'ayant pas de désir de grossesse utilisaient régulièrement le préservatif $(73,1)$ que ceux qui avaient le désir d'enfants $(61,1 \%)$.

Dans les foyers où il existait une différence de statut sérologique, les partenaires négatifs $(67,3 \%)$ faisaient l'usage régulier du préservatif que ceux qui sont positifs $(54,2 \%)$

Tableau II : usage régulier du préservatif dans les rapports sexuels intraconjugaux durant les 6 derniers mois

\begin{tabular}{|c|c|c|c|c|c|}
\hline Variable & & $\begin{array}{l}\text { Port régulier } \\
\text { du préservatif }\end{array}$ & $\begin{array}{l}\text { Port irrégulier } \\
\text { du préservatif }\end{array}$ & $\begin{array}{l}\text { Effectif de la } \\
\text { variable étudiée }\end{array}$ & $P(a=5 \%)$ \\
\hline \multirow{2}{*}{$\begin{array}{l}\text { Situation } \\
\text { maritale } \\
\mathbf{N = 8 1}\end{array}$} & Célibataire & $\begin{array}{l}22 \\
(75,9)\end{array}$ & $\begin{array}{l}7 \\
(24,1)\end{array}$ & $\begin{array}{l}29 \\
(35,8)\end{array}$ & \multirow{2}{*}{$<0,30$} \\
\hline & $\begin{array}{l}\text { Vie en } \\
\text { couple* }\end{array}$ & $\begin{array}{l}32 \\
(61,5)\end{array}$ & $\begin{array}{l}20 \\
(38,5)\end{array}$ & $\begin{array}{l}52 \\
(64,2)\end{array}$ & \\
\hline \multirow{2}{*}{$\begin{array}{l}\text { Statut } \\
\text { sérologique } \\
\text { du partenaire } \\
\text { sexuel } \\
\mathbf{N}=62\end{array}$} & Négatif & $\begin{array}{l}24 \\
(67,3)\end{array}$ & $\begin{array}{l}14 \\
(36,7)\end{array}$ & $\begin{array}{l}38 \\
(61,3)\end{array}$ & \multirow{2}{*}{$<0,50$} \\
\hline & Positif & $\begin{array}{l}13 \\
(54,2)\end{array}$ & $\begin{array}{l}11 \\
(45,8)\end{array}$ & $\begin{array}{l}24 \\
(38,7)\end{array}$ & \\
\hline $\begin{array}{l}\text { Partage du } \\
\text { statut } \\
\text { sérologique } \\
\text { avec le } \\
\text { partenaire }\end{array}$ & Oui & $\begin{array}{l}39 \\
(63,9)\end{array}$ & $\begin{array}{l}22 \\
(36,1)\end{array}$ & $\begin{array}{l}61 \\
(77,2)\end{array}$ & \multirow[t]{2}{*}{$<0,90$} \\
\hline$N=79$ & Non & $\begin{array}{l}11 \\
(61,1)\end{array}$ & $\begin{array}{l}7 \\
(38,9)\end{array}$ & $\begin{array}{l}18 \\
(22,8)\end{array}$ & \\
\hline
\end{tabular}




\begin{tabular}{|c|c|c|c|c|c|}
\hline \multirow[t]{2}{*}{$\begin{array}{l}\text { Nombre } \\
\text { d'enfants du } \\
\text { patient } \mathbf{N}=\mathbf{8 3}\end{array}$} & $\begin{array}{l}\text { Patient sans } \\
\text { enfant }\end{array}$ & $\begin{array}{l}9 \\
(50)\end{array}$ & $\begin{array}{l}9 \\
(50)\end{array}$ & $\begin{array}{l}18 \\
(21,7)\end{array}$ & \multirow[b]{2}{*}{$<0,20$} \\
\hline & $\begin{array}{l}\text { Patient avec } \\
\text { un enfant et } \\
\text { plus }\end{array}$ & $\begin{array}{l}44 \\
(67,7)\end{array}$ & $\begin{array}{l}21 \\
(32,3)\end{array}$ & $\begin{array}{l}65 \\
(78,3)\end{array}$ & \\
\hline \multirow[t]{2}{*}{$\begin{array}{l}\text { Désir } \\
\text { d'enfant } \\
\mathbf{N}=\mathbf{8 0}\end{array}$} & $\begin{array}{l}\text { Désir } \\
\text { d'enfants }\end{array}$ & $\begin{array}{l}33 \\
(61,1)\end{array}$ & $\begin{array}{l}21 \\
(38,9)\end{array}$ & $\begin{array}{l}54 \\
(67,5)\end{array}$ & \multirow[t]{2}{*}{$<0,30$} \\
\hline & $\begin{array}{l}\text { Pas de désir } \\
\text { d'enfants }\end{array}$ & $\begin{array}{l}19 \\
(73,1)\end{array}$ & $\begin{array}{l}7 \\
(26,9)\end{array}$ & $\begin{array}{l}26 \\
(32,5)\end{array}$ & \\
\hline \multirow{2}{*}{$\begin{array}{l}\text { Nombre } \\
\text { d'années de } \\
\text { suivi dans les } \\
\text { soins } \\
\mathbf{N = 8 5}\end{array}$} & $\begin{array}{l}\text { Suivi moins } \\
\text { de } 3 \text { ans }\end{array}$ & $\begin{array}{l}18 \\
(64,3)\end{array}$ & $\begin{array}{l}10 \\
(35,7)\end{array}$ & $\begin{array}{l}28 \\
(32,9)\end{array}$ & \multirow[b]{2}{*}{$<0,95$} \\
\hline & $\begin{array}{l}\text { Suivi plus } \\
\text { de } 3 \text { ans }\end{array}$ & $\begin{array}{l}36 \\
(63,2)\end{array}$ & $\begin{array}{l}21 \\
(36,8)\end{array}$ & $\begin{array}{l}57 \\
(67,1)\end{array}$ & \\
\hline \multirow{2}{*}{$\begin{array}{l}\text { Acceptation } \\
/ \text { Tolérance du } \\
\text { port du } \\
\text { préservatif } \\
\mathbf{N}=\mathbf{8 3}\end{array}$} & Non & $\begin{array}{l}14 \\
(77,8)\end{array}$ & $\begin{array}{l}4 \\
(22,2)\end{array}$ & $\begin{array}{l}18 \\
(21,7)\end{array}$ & \multirow[b]{2}{*}{$<0,20$} \\
\hline & Oui & $\begin{array}{l}39 \\
(60)\end{array}$ & $\begin{array}{l}26 \\
(40)\end{array}$ & $\begin{array}{l}65 \\
(78,3)\end{array}$ & \\
\hline $\begin{array}{l}\text { Perception de } \\
\text { l'usage du } \\
\text { préservatif } \\
\text { comme } \\
\text { néfastes pour } \\
\text { la santé } \\
\mathbf{N = 8 0}\end{array}$ & Non & $\begin{array}{l}15 \\
(78,9)\end{array}$ & $\begin{array}{l}4 \\
(21,1)\end{array}$ & $\begin{array}{l}19 \\
(23,8)\end{array}$ & $<0,20$ \\
\hline
\end{tabular}

\section{Discussion}

Les résultats de l'étude ont montré que le dépistage du VIH chez les patients interrogés n'avait pas mis un terme à leur relation sexuelle. Ainsi, les rapports sexuels dans le foyer continuent d'être pratiqués chez $72 \%$ des personnes interrogées. Le préservatif introduit dans la relation conjugale est utilisé mais pas régulièrement. L'usage n'était régulièrement constant que chez 53,5\% des personnes. Ce taux était identique à ceux rapportés par Yaba et al., (2013) et Deuba et al., (2018).

L'étude a identifié des facteurs qui ont influencé l'usage du préservatif. Ainsi dans le foyer conjugal, le fait d'être célibataire ou d'être informé du statut partenaire ou d'avoir une différence de statut étaient plus associés à 
l'utilisation constante du préservatif. Cependant, tous ces facteurs ne se sont pas révélés significativement liés à une utilisation régulièrement constante du préservatif. Par contre, avoir le désir d'enfants ou être de même statut sérologique que le partenaire étaient plutôt associés à une utilisation non constante du préservatif mais le lien n'était pas significatif. Enfin la durée du suivi dans les soins n'avait pas affecté l'usage constant ou non constant du préservatif. Au total aucune des variables étudiées n'est associée statistiquement significative à un usage régulièrement constant du préservatif. Si l'on fait un raisonnement par analogie avec le sexe sans condom qui serait lié à certains nombres de paramètres tels que la non divulgation du statut entre partenaire, l'absence du dépistage du partenaire, la discrimination et la stigmatisation (Wagner et al. , 2016); (Yaba et al., 2013), l'on pourrait prédire une forte utilisation du préservatif dans cette étude puisque sur tous les sites de prise en charge des actions comme le partage du statut entre partenaire, le dépistage du couple, la lutte contre la discrimination et la stigmatisation sont menées pour une meilleur insertion sociale des personnes vivant avec le VIH surtout qu'au niveau national, le niveau de discrimination et de stigmatisation a globalement diminué et les taux dépistage et de partage du statut entre partenaire sexuel se sont améliorés dans le pays (PNLS, 2019). Cependant l'on doit être prudent, l'amélioration de ces indicateurs ne corroborent pas forcement à l'utilisation du préservatif. En effet lorsque le partage du statut sérologique au sein du couple est largement fait et que la proportion de statut seroconcordant est élevée, les rapports sexuels non protégés deviennent fréquents comme l'ont fait remarqué Chakrapani et al., (2010) ; Cook et al.(2018) ; Fetene et al., (2018) et Tadesse et al., (2019) dans les résultats de leurs études. Mais l'usage du préservatif semble être plus complexe et ne peut être associé voire lié qu'à ces paramètres étudiés.

En effet, l'usage régulier ou irrégulier du préservatif semble être déterminé par les circonstances selon lesquelles les problèmes et les besoins du couple évoluent (Schiltz et al., 1999). Ces circonstances peuvent prendre des significations très différentes et répondent à des logiques particulières (Musinguzi et al. , 2015).

En matière de prévention, les personnes les plus concernées tentent de rétablir un certain équilibre en intégrant et en adaptant les consignes de prévention à partir de leurs besoins, de leur désir mais aussi de leurs difficultés. Schroder et al., en 2007 ont montré l'influence de l'humeur sur le port du préservatif. En effet en 1999, Schiltz et al., écrivaient que pour les personnes vivant avec le VIH qui retrouvent une existence où le temps de vivre et le bienêtre reprennent leurs droits, la question de la prévention est centrale. Ces personnes conçoivent des stratégies de réduction des risques qui auront leur propre rationalité et qui ne seront pas forcément conformes aux normes établies. 
Il ne s'agit pas pour la personne infectée de faire délibérément courir un risque à son partenaire sexuel, mais il s'agit plutôt d'un processus d'adaptation et d'arbitrage du couple face à plusieurs risques (Bunnell et al.,2005) dont les résultats sont instables, et au centre duquel vont jouer plusieurs paramètres : le désir d'enfant, l'état de santé, le niveau d'influence du sujet dans le foyer, le vœu de partager la vie de l'autre, la crainte de la solitude, le mode de vie. D'où le maintien rigoureux et constant d'un comportement protecteur devient très difficile (Delor et al., 1998 ; Schroder et al., 2007 ; Reis\& Gir, 2010) et la prise de risque devient fréquente ce qui justifie l'usage irrégulier du préservatif chez ces personnes conscientes de la transmission du VIH à travers des conseils de prévention qu'elles reçoivent sur les sites de prise en charge. Ainsi, protéger son ( $\mathrm{sa}$ ) partenaire contre le VIH place l'homme et la femme dans des situations différentes. Et chacun selon son pouvoir d'influence dans le ménage impose sa volonté (Reis\& Gir, 2010).

Dans cette étude, l'usage régulier du préservatif était meilleur dans le couple où le client interrogé est un homme $(78,6 \%)$ qu'une femme $(56,1 \%)$. L'influence du sexe masculin sur l'usage régulier de ce dispositif de barrière se justifie : primo sur les sites se sont les préservatifs masculins qui étaient distribués, secundo parce qu'il est généralement porté par l'homme. Alors sa négociation semble être d'autant plus étroite lorsque la femme se trouve en situation de vulnérabilité sociale et économique (Toska et al., 2017). L'usage du préservatif peut être décidé unilatéralement ou imposé par un des partenaires et cela donnerait une mauvaise perception de son utilisation comme c'était le cas dans cette étude pour $21,2 \%$ des patients interviewés qui en faisaient usage contre leur gré. Mais selon les auteurs Schiltz et al., (1999); Myer et al., ( 2002) ; Ngure et al., (2012) ; Deuba et al., (2018) plusieurs logiques prévalent à l'intérieur des couples stables où la question de la protection du partenaire constitue un thème particulièrement problématique qui s'étend non seulement à l'utilisation du préservatif mais encore à l'incertitude qui entoure un large éventail de conduites érotiques, comme par exemples:

-Dans un foyer où un des partenaires n'a pas un bon état de santé, l'usage systématique de préservatif peut être la règle pour une prévention de l'infection chez la personne négative. Ce qui ne reflète plus la situation actuelle. Aujourd'hui, en Côte d'Ivoire, avec l'accès au traitement ARV, le PNLS (2019) a rapporté un recul de la morbidité chez ces patients et une amélioration de leur état de santé ce qui pourrait expliquer le relâchement de l'usage du préservatif.

-Aussi, le désir d'enfants exprimés par 62\% des patients justifierait une utilisation irrégulière du préservatif car un couple qui désirait d'enfants trouverait absurde de réaliser ce rêve en faisant un usage régulier de ce dispositif de barrière qui l'empêcherait d'enfanter (Sarkar, 2008). 
-A l'extrême, l'abandon de toute protection peut être permanent et accepté par les deux partenaires parfaitement informés de la présence du VIH dans leur relation. Dans ce cas deux situations peuvent expliquer l'abandon du port du préservatif : soit le couple recherche un compromis plus ou moins acceptable avec un des partenaires qui supporte mal les frustrations associées à l'usage du préservatif car l'image du préservatif peut être mal perçu par le partenaire dans les actes sexuels (Exaveryal., 2012) comme c'est le cas dans l'étude pour $22,8 \%$ des sujets interrogés. Ou soit le couple manifeste un idéal d'amour dans une relation sexuelle sans barrière, sans contrainte comme le stipule l'adage du mariage : « unis pour le bonheur et le pire ». Cela traduit le désir de privilégier la qualité de la relation amoureuse et de négliger les risques de transmission et de surinfections.

Aujourd'hui, les connaissances des populations sur l'infection par le VIH ont évolué et l'usage du préservatif suivant certainement cette évolution, semble être dépassé. En effet, concernant les facteurs influençant la prise de risque, le traitement antiretroviral n'est pas épargné comme Sarna et al., (2009) et Liu et al., (2014) ont fait remarquer dans les études. En effet, le traitement antiretroviral hautement actif a modifié les attitudes des personnes vivant avec le VIH dans le sens d'une plus grande prise de risque. Tous ces patients interrogés dans cette étude sont sous des traitements antiretroviraux. Ces personnes ne se sentaient plus comme des malades se donneraient à des comportements et de pratiques sexuelles libres (Tadesse et al., 2019). Ainsi le préservatif semble être abandonné ou son usage dans le foyer devenait plus occasionnel que régulier.

\section{Conclusion}

Les données de cette étude sont issues exclusivement des déclarations des patients qui sont suivis sur un site de prise en charge où les conseils de prévention sont dispensés. En plus, les informations recueillies étaient plus en rapport avec l'intimité des patients. A cet effet la sincérité de ces déclarations ne peut être prouvée. Cependant les résultats obtenus sont superposables à ceux trouvés par d'autres auteurs cités dans cette étude.

Les résultats de l'étude ont montré que les personnes vivant avec VIH et éduquées au port de préservatif affirment l'introduire dans leur relation sexuelle intraconjugale. Mais son usage régulier est émaillé de nombreuses difficultés.

Les facteurs individuels, de situation matrimoniale, de reproduction, de dépistage, de divulgation du statut, de comportement sexuel dans le foyer, d'acceptabilité du préservatif qui y sont associés, ne sont pas statistiquement liés à l'usage régulier du préservatif. C'est ce qui expliquerait la difficulté de synthèse des études qui s'attachent à isoler les déterminants liés à l'usage systématique du préservatif. 
Néanmoins, l'usage du préservatif serait plutôt déterminé par les problèmes et les besoins des individus qui l'utilisent. La difficulté de son utilisation régulière résulterait de la complexité et de la variabilité de ces problèmes et besoins vécus ou ressentis par les usagers mais aussi de la perception et la représentation de la prévention de la transmission sexuelle par les personnes infectées par le VIH sous traitement antiretroviral.

Des études plus poussées sur les besoins de sexualité des personnes vivant avec le VIH permettront de mieux établir des liens pour l'usage régulier du préservatif.

\section{References:}

1. Bunnell, R.E., Nassozi J., Marum, E., Mubangizi, J., Malamba, S., Dillon, B., et al., (2005). Living with discordance: knowledge, challenges, and prevention strategies of HIV-discordant couples in Uganda. AIDS Care.

2. Chakrapani, V., Newman, P. A., Shunmugam, M., \&Dubrow, R. (2010). Prevalence and contexts of inconsistent condom use among heterosexual men and women living with HIV in India: implications for prevention. AIDS patient care and STDs, 24(1), 49-58. doi:10.1089/apc.2009.0214

3. Cook, C. L., Staras, S., Zhou, Z., Chichetto, N., \& Cook, R. L. (2018). Disclosure of HIV serostatus and condomless sex among men living with HIV/AIDS in Florida. PloS one, 13(12), e0207838. doi:10.1371/journal.pone.0207838

4. Delor, F. et Schiltz, M.A. (1998). Séropositifs. Trajectoires identitaires et rencontres du risque. In: Sciences sociales et santé. Volume 16, n4, pp. 107-111. [visité le 10/06/2019]. En ligne : https://www.persee.fr/doc/sosan_0294-0337_1998_num_16_4_1780

5. Deuba, K., Kohlbrenner, V., Koirala, S., Ekström, A. M., \& CAT-S group (2018). Condom use behaviour among people living withHIV: a seven-country community-based participatory research in the AsiaPacific region. Sexually transmitted infections, 94(3), 200-205. doi:10.1136/sextrans-2017-053263Direction de l'Informatique et de l'Information Sanitaire (DIIS). (2018). Rapport annuel VIH 2017. Abidjan, Côte d'Ivoire : Ministère de la santé et de l'hygiène publique (MSPH)

6. Exavery, A., Kanté, A.M., Jackson, E., Noronha, J., Sikustahili, G., Tani, K., Mushi, H.P., Baynes, C., Ramsey, K. \& Hingora A., Phillips JF. (2012). Role of condom negotiation on condom use among women of reproductive age in three districts in Tanzania. BMC PublicHealt, 20;12:1097. doi: 10.1186/1471-2458-12-1097. 
7. Liu, H., Su Y, Zhu, L., Xing, J., Wu, J. \& Wang, N. (2014). Effectiveness of ART and condom use for prevention of sexual HIV transmission in serodiscordant couples: asystematicreview and metaanalysis. PLoS One, 4;9(11):e111175. doi:10.137.

8. Musinguzi, G., Bastiaens, H., Matovu, J. K., Nuwaha, F., Mujisha, G., Kiguli, J., ... Wanyenze, R. K. (2015). Barriers to Condom Use among High Risk Men Who Have Sexwith Men in Uganda: A Qualitative Study. PloS one, 10(7), e0132297. doi:10.1371/journal.pone.0132297

9. Myer, L., Mathews, C., \& Little, F. (2002). Condom use and sexual behaviors among individuals procuring free male condoms in South Africa: a prospective study. Sex Transm Dis., 29(4):239-41.

10. Ngure, K., Mugo, N., Celum, C., Baeten, JM, Morris, M, Olungah, O, Olenja, J, Tamooh, H, \& Shell-Duncan, B. (2012). A qualitative study of barriers to consistent condom use among HIV-1 serodiscordant couples in Kenya. AIDS Care, 24(4):509-16. doi:10.1080/09540121.2011.613911.

11. Onayade, A.A., Abiona, T.C., Ugbala, C., Alozie G., Adetuyi, O. (2008). Determinants of consistent condom use among adolescents and young adults attending a tertiary educational institution in Ile-Ife, Nigeria. Niger Postgrad Med J. , 15(3):185-91.

12. Programme commun des Nations Unies sur le VIH/sida (ONUSIDA). (2017). En finir avec le sida; progresser vers les cibles 90-90-90. Genève, Suisse : ONUSIDA. [Visité le 26/12/2018]. En ligne https://www.unaids.org/sites/default/files/media_asset/20170720_enfinir-avec-le-sida_synthese.pdf.

13. Programme National de Lutte contre le Sida (PNLS). (2019). Analyse de la situation épidémiologique et de la réponse au VIH en Côte d'Ivoire. Revue à mi-parcours du PSN 2016-2020. Abidjan, Côte d'Ivoire: Ministère de la santé et de l'hygiène publique (MSPH)

14. Reis RK, Gir E. (2010). Living with the difference: the impact of serodiscordance on the affective and sexual life of HIV/AIDS patients. Rev Esc Enferm USP.

15. Sarna, A., Chersich, M., Okal, J., Luchters, S.M.F., Mandaliya, N.K., Rutenberg et al. (2009). Changes in sexual risk taking with antiretroviral treatment: influence of context and gendernorms in Mombasa, Kenya, Culture, Health\&Sexuality, 11:8, 783-797, DOI: 10.1080/13691050903033423

16. Sarkar, N.N. (2008). Barriers to condom use. Eur J Contracept ReprodHealth Care. 2008; 13(2):114-122

17. Schiltz, M.A., Meystre-Agustoni, G., Delor, F, Pierret, J, Adam, P., Bouhnik, A.D. et al. (1999). Vie sexuelle et Prises de risques chez les 
personnes vivant avec le VIH/sida. Dans Collection Sciences sociales et Sida (dir.)

18. Séropositivité, vie sexuelle et risque de transmission du VIH. Paris, France : Agence Nationale de Recherches sur le Sida (ANRS); 72p112p [visité le 12/08/2018]. En ligne : https://serval.unil.ch/resource/serval:BIB_7174.P001/REF.pdf

19. Schroder, K.E., et Johnson, C.J., Wiebe, J.S. (2007 Apr). An eventlevel analysis of condom use as a function of mood, alcohol use, and safer sex negotiations. Arch SexBehav.;38(2):283-9.

20. Tadesse, W. B., \& Gelagay, A. A. (2019). Risky sexual practice and associated factorsamong HIV positive adults visiting ART clinics in public hospitals in Addis Ababa city, Ethiopia: a cross sectional study. BMC public health, 19(1), 113. doi:10.1186/s12889-019-6438-5

21. Toska, E., Pantelic, M., Meinck, F., Keck, K., Haghighat, R., Cluver, L. (2017). Sex in the shadow of HIV: A systematic review of prevalence, riskfactors, and interventions to reduce sexual risk-taking among HIV-positive adolescents and youth in sub-Saharan Africa. PLoS One. 2017;12(6):e0178106. Published 2017 Jun 5. doi:10.1371/journal.pone.0178106

22. Wagner, G. J., Bogart, L. M., Klein, D. J., Green, H. D. Jr., Mutchler, M. G., McDavitt, B., \& Hilliard, C. (2016). Association of Internalized and Social Network Level HIV Stigma With High-Risk Condomless Sex Among HIV-Positive African American Men. Archives of sexual behavior, 45(6), 1347-1355. doi:10.1007/s10508-015-0641-y

23. Yaba, W., Msellati, P. et Chippaux, J.P. (2013). Comportements sexuels et usage du préservatif chez les personnes vivant avec le VIH/sida au Gabon. Santé Publique, (Vol. 25), p. 839-847. DOI $10.3917 /$ spub.136.0839 